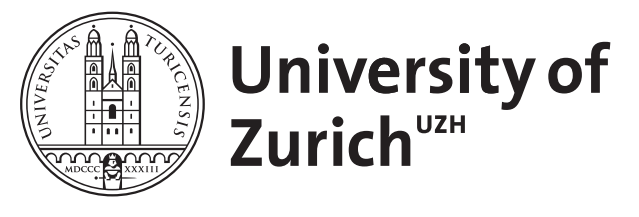

\title{
Chemotherapie von Hirntumoren bei Erwachsenen
}

\author{
Roth, P ; Weller, M
}

\begin{abstract}
The treatment of patients with brain tumors has long been the domain of neurosurgery and radiotherapy but chemotherapy is now well established as an additional treatment option for many tumor entities in neuro-oncology. This is particularly true for patients with newly diagnosed and relapsing glioblastoma and anaplastic glioma as well as the treatment of medulloblastoma and primary lymphoma of the central nervous system (CNS). In addition to purely histopathological features, treatment decisions including those for chemotherapy are now based increasingly more on molecular tumor profiling. Within the group of gliomas these markers include the methylation status of the O-6-methylguanine-DNA methyltransferase (MGMT) promoter and the 1p/19q status, which reflects the loss of genetic material on chromosome arms $1 \mathrm{p}$ and $19 \mathrm{q}$. The presence of a $1 \mathrm{p} / 19 \mathrm{q}$ codeletion is associated with a better prognosis and increased sensitivity to alkylating chemotherapy in patients with anaplastic gliomas.
\end{abstract}

DOI: https://doi.org/10.1007/s00115-014-4242-0

Other titles: Chemotherapy of brain tumors in aduts

Posted at the Zurich Open Repository and Archive, University of Zurich

ZORA URL: https://doi.org/10.5167/uzh-110463

Journal Article

Accepted Version

Originally published at:

Roth, P; Weller, M (2015). Chemotherapie von Hirntumoren bei Erwachsenen. Der Nervenarzt, 86(4):495508.

DOI: https://doi.org/10.1007/s00115-014-4242-0 


\section{Chemotherapie von Hirntumoren des Erwachsenenalters}

Patrick Roth $^{1 *}$, Michael Weller ${ }^{1}$

${ }^{1}$ Klinik für Neurologie und Hirntumorzentrum, UniversitätsSpital Zürich

*Korrespondenz: PD Dr. Patrick Roth, Klinik für Neurologie, UniversitätsSpital Zürich, Frauenklinikstrasse 26, 8091 Zürich, Tel.: +41 (0)44 255 5511, Fax: +41 (0)44 255 4380, Email: patrick.roth@usz.ch

Abbkürzungen: Ara-C, Cytarabin; AWMF, Arbeitsgemeinschaft der Wissenschaftlichen Medizinischen Fachgesellschaften e.V; BRAF, v-raf murine sarcoma viral oncogene homolog B; CTLA-4, cytotoxic T lymphocyte-associated protein 4; EORTC, European Organisation for Research and Treatment of Cancer; EGFR, Rezeptor des epidermalen Wachstumsfaktors; MGMT, $\mathrm{O}^{6}$-Methylguanin-DNA-Methyltransferase, mTOR, mechanistic target of rapamycin; MTX, Methotrexat; NOA, Neuroonkologische Arbeitsgemeinschaft; PCNSL, primäre Lymphome des Zentralnervensystems; PD-1, programmed cell death protein 1; PDGF, platelet-derived growth factor; PNET, primitive neuroektodermale Tumoren; RTOG, Radiation Therapy Oncology Group; SEGA, subependymal giant cell astrocytoma; SHH, sonic hedgehog; SMO, smoothened receptor; TSC, tuberous sclerosis complex; VEGF, vascular endothelial growth factor; WHO, World Health Organization. 


\section{Zusammenfassung}

Die Behandlung von Hirntumorpatienten war lange Zeit eine Domäne der Neurochirurgie und Strahlentherapie. Mittlerweile ist für viele Tumorentitäten in der Neuroonkologie die Chemotherapie als zusätzliche Therapieoption fest etabliert. Dies gilt insbesondere für die Primär- und Rezidivtherapie von Glioblastomen und anaplastischen Gliomen, die Behandlung von Medulloblastomen und ZNS-Lymphomen sowie teilweise bei Patienten mit Hirnmetastasen.

Neben der reinen histopathologischen Einordnung erfolgt die Indikationsstellung für eine chemotherapeutische Behandlung mittlerweile auch zunehmend basierend auf dem molekularen Tumorprofil. Hierzu zählen bei den Gliomen insbesondere der Methylierungsstatus des MGMT-Promotors sowie der 1p/19q-Status, der den Verlust genetischen Materials auf den Chromosomenarmen 1p und 19q beschreibt. Das Vorhandensein einer 1p/19q-Kodeletion geht bei anaplastischen Gliomen mit einer besseren Prognose und einer erhöhten Empfindlichkeit gegenüber alkylierender Chemotherapie einher. Stark limitiert bleiben die chemotherapeutischen Behandlungsoptionen bei Meningeomen, Schwannomen und Neurinomen. Auch für Ependymome stehen keine etablierten medikamentösen Therapieoptionen zur Verfügung. Bei Hirnmetastasen orientiert sich die Wahl des Chemotherapeutikums an den Behandlungskonzepten für den jeweiligen Primärtumor. Molekulare Veränderungen, z.B. das Vorhandensein von Mutationen im BRAFGen, sind auch hier hilfreich, Patienten zu identifizieren, die von gezielten Therapieversuchen mit entsprechenden Inhibitoren profitieren können. Die Meningeosis neoplastica muss in Abhängigkeit von weiteren soliden Tumormanifestationen in- und ausserhalb des ZNS mit Strahlentherapie, systemischer Chemotherapie oder einer intrathekalen Chemotherapie behandelt werden.

Schlüsselwörter: Hirntumor, Chemotherapie, Glioblastom, MGMT, 1p/19q 


\section{Summary}

The treatment of patients with brain tumors has long been the domain of neurosurgery and radiotherapy. Meanwhile, chemotherapy is well established as an additional treatment option for many tumor entities in neuro-oncology. This is particularly true for patients with newly diagnosed and relapsed glioblastoma and anaplastic gliomas as well as the treatment of medulloblastomas and primary CNS lymphomas.

In addition to pure histopathological features, treatment decisions including those for chemotherapy are now increasingly based on the molecular tumor profiling. Within the group of gliomas, these markers include the methylation status of the MGMT promoter and the $1 \mathrm{p} / 19 \mathrm{q}$ status which reflects the loss of genetic material on chromosome arms $1 \mathrm{p}$ and $19 \mathrm{q}$. The presence of a 1p/19q co-deletion is associated with better prognosis and increased sensitivity to alkylating chemotherapy in patients with anaplastic gliomas.

Chemotherapeutic treatment options for meningiomas, schwannomas and neurinomas remain very limited. Similary, chemotherapy is not well established for patients with relapsed ependymoma. The selection of a chemotherapy protocol for patients with brain metastases is mainly based on the general treatment concept for the corresponding primary tumor.

Molecular alterations such as the presence of mutations in the BRAF or EGFR gene are useful to identify patients who may respond to targeted therapies using "small molecule inhibitors" or antibodies. Dissemination of tumor cells into the cerebrospinal fluid is a particular challenge. Depending on the presence of other tumor manifestations in the brain parenchyma or outside the CNS, radiotherapy, systemic chemotherapy or intrathecal chemotherapy must be considered.

Key words: Brain tumor, chemotherapy, glioblastoma, MGMT, 1p/19q 


\section{Darstellung des Lernziels}

Die medikamentöse Therapie von Hirntumoren hat in den letzten Jahren einige Veränderungen erfahren. Nach der Lektüre dieses Artikels kennen Sie...:

- Bedeutung und Stellenwert der Chemotherapie bei primären Hirntumoren

- Unterschiedliche chemotherapeutische Konzepte bei Erstdiagnose und Rezidiv

- Indikationen für eine chemotherapeutische Behandlung bei Hirnmetastasen und Meningeosis neoplastica

- Neue vielversprechende Therapieansätze, die sich aktuell in der klinischen Entwicklung befinden

\section{Einführung}

In diesem Beitrag werden Therapiestandards und neue Entwicklung in der medikamentösen Primär- sowie Rezidivtherapie für die häufigsten primären Hirntumoren sowie für Hirnmetastasen und die Meningeosis neoplastica im Erwachsenenalter aufgezeigt. Neben den seit langer Zeit etablierten Therapieformen der Operation und der Strahlentherapie hat sich die medikamentöse Tumortherapie insbesondere bei den primären Hirntumoren in den letzten 10 Jahren als weitere Therapieoption fest etabliert. Dieser Beitrag fasst die wichtigsten Entwicklungen der medikamentösen Therapie von Hirntumoren der letzten Jahre zusammen mit einem Schwerpunkt auf praxisrelevante Veränderungen, die in den meisten Fällen auf den Ergebnissen grosser Studien basieren. Die aufgeführten Therapieempfehlungen und Einschätzungen sind weitgehend kongruent mit den Therapieleitlinien der Deutschen Gesellschaft für Neurologie und der Neuro-Onkologischen Arbeitsgemeinschaft (NOA) in der Deutschen Krebsgesellschaft (http://www.awmf.org/leitlinien.html ).

Neben der am häufigsten eingesetzten systemischen Therapie spielen die intratumorale bzw. 
intrathekale Administration von Chemotherapeutika nur eine untergeordnete Rolle bei einigen wenigen Krankheitsbildern. Fest etabliert ist die Chemotherapie insbesondere in der Primärund Rezidivtherapie der anaplastischen Gliome und Glioblastome, bei primären ZNSLymphomen sowie bestimmten Keimzelltumoren. Neben den klassischen Chemotherapeutika, die u.a. Alkylantien, Inhibitoren der Topoisomerase I und II sowie Antimetabolite umfassen, sind Angiogeneshemmer und Tyrosinkinaseinhibitoren in den letzen Jahren intensiv untersucht worden (Tabelle 1). Noch nicht Teil der Standardtherapie aber teilweise in fortgeschrittener klinischer Entwicklung sind verschiedene immuntherapeutische Behandlungsansätze, die in Zukunft hoffentlich eine weitere Behandlungsoption für verschiedene Tumorentitäten darstellen werden. Neben der eigentlichen tumorspezifischen Therapie stehen im Bereich der Neuroonkologie zudem verschiedene medikamentöse Therapieansätze zur Verfügung, welche supportiv wirken und zum Ziel haben, die Lebensqualität aufrechtzuerhalten bzw. zu verbessern. Hierzu zählen insbesondere Glucocorticoide wie Dexamethason sowie in vielen Fällen Antikonvulsiva. Diesbezüglich wird auf die weiterführende Literatur verwiesen [1, 2].

\section{Molekulare Marker mit Einfluss auf die Therapieentscheidung}

Die Entscheidung über den Einsatz der Chemotherapie bei primären Hirntumoren wird immer häufiger vom molekularen Tumorprofil abhängig gemacht. Dies gilt insbesondere für die Behandlung im Rahmen klinischer Studien, aber zunehmend auch im klinischen Alltag. Von besonderer Bedeutung sind hierbei der Methylierungsstatus des $\mathrm{O}^{6}$-Methylguanin-DNAMethyltransferase (MGMT)-Promotors, der 1p/19q-Status und das Vorhandensein von Mutation in den Isozitratdehydrogenase (IDH)-Genen. Mutationen im IDH1 oder IDH2-Gen gehen bei Gliomen mit einer besseren Prognose einher [3]. Ein klarer prädiktiver Wert für das Ansprechen auf Strahlen- oder Chemotherapie ist aus dem IDH-Status bis jetzt aber noch nicht ableitbar. Im Gesatz dazu können der MGMT- und 1p/19q-Status bei bestimmen 
Patientengruppen für therapeutische Entscheidungen herangezogen werden. Das MGMT-Gen kodiert für ein Protein, das Alkylgruppen von der DNA entfernt und somit den Effekt des Chemotherapeutikums Temozolomid (möglicherweise auch anderer, ähnlich wirkender Substanzen), revertiert. In Glioblastomen geht vermutlich die Expression von MGMT durch eine Methylierung des Promotors bei ca. 30-40\% aller Patienten verloren. Das Fehlen dieses Reperaturproteins bedingt eine Sensitivität gegenüber alkylierender Chemotherapie mit Temozolomid. Ob dieses Phänomen alleine durch die Methylierung des MGMT-Promotors erklärt wird, oder ob der MGMT-Status eher im Rahmen einer generellen Veränderung epigenetischer Genregulation betrachtet werden muss, ist derzeit Gegenstand wissenschaftlicher Untersuchungen und hängt vermutlich wesentlich von der Frage des IDHStatus ab [4]. Die Bestimmung des MGMT-Methylierungsstatus ist mit verschiedenen Techniken möglich und sollte in einem Labor mit entsprechender Expertise erfolgen. Unter dem 1p/19q-Status wird der kombinierte Verlust genetischer Information auf dem kurzen Arm von Chromsom 1 (1p) bzw. des langen Arms von Chromosom 19 (19q) verstanden. Ursächlich liegt eine unbalancierte Translokation der Chromosomen 1 und 19 zugrunde. Patienten mit anaplastischen Gliomen mit 1p/19q-Kodeletion haben eine bessere Prognose als Patienten, deren Tumor keine solche Veränderung aufweist. Darüber hinaus hat das Vorliegen einer 1p/19q-Kodeletion einen prädiktiven Wert für das Ansprechen auf eine kombinierte Radiochemotherapie bei anaplastischen Gliomen (s.u.). Auch für die Bestimmung des 1p/19qStatus stehen verschiedene Methoden zur Verfügung. 


\section{Spezifische Empfehlungen zum Einsatz der Chemotherapie bei einzelnen}

\section{Tumorentitäten}

\section{Gliome}

\section{Glioblastome}

Seit 2005 ist die Chemotherapie in Form des Alkylans Temozolomid fixer Bestandteil der Standardtherapie von Patienten mit einem neu diagnostizierten Glioblastom. Ausnahmen bestehen insbesondere bei älteren Patienten (s.u.) sowie bei Patienten in eingeschränktem Allgemeinzustand. In der Primärtherapie wird Temozolomid in einer Dosierung von 75 $\mathrm{mg} / \mathrm{m}^{2}$ konkomitant zur Strahlentherapie sowie im Anschluss für bis zu 6 Zyklen Erhaltungstherapie mit $150-200 \mathrm{mg} / \mathrm{m}^{2}$ an 5 von 28 Tagen verabreicht [5]. Eine Intensivierung der Dosis war dem Standardschema in einer randomisierten Phase III-Studie nicht überlegen [6]. Subgruppenanalysen grösser Studien deuten zudem darauf hin, dass eine Verlängerung der Zyklenzahl der Erhaltungstherapie keinen Vorteil bringt. Die prädiktive Bedeutung des MGMT-Promotorstatus für ein Ansprechen auf Temozolomid wurde zwischenzeitlich an mehreren grossen Studienkollektiven bestätigt [7]. Da für Patienten mit einem unmethylierten MGMT-Promotor keine sinnvollen Alternativen zu Temozolomid zur Verfügung stehen, die MGMT-Testung nicht standardisiert ist und auch Patienten mit einem unmethylierten MGMT-Promotor von Temozolomid profitieren können, wird die Therapie in der Regel unabhängig vom MGMT-Status durchgeführt. Die Hinzunahme des VEGFAntikörpers Bevacizumab zur Temozolomid-basierten Radiochemotherapie verlängert das progressionsfreie Überleben, nicht aber das Gesamtüberleben [8, 9]. Basierend auf diesen Phase III-Studiendaten besteht bis jetzt, und mutmasslich auch in der Zukunft, keine Zulassung für Bevacizumab in der Primärtherapie. Auch der Integrin-Inhibitor Cilengitide blieb in einer Phase III-Studie bei Patienten mit einem neu diagnostizierten Glioblastom 
wirkungslos [10].

Fast alle Patienten mit einem Glioblastom erleiden im Verlauf ein Tumorrezidiv. Ein einheitlicher Therapiestandard existiert nicht. Neben einer erneuten Resektion und - in seltenen Fällen - einer nochmaligen Bestrahlung kommen in erster Linie medikamentöse Therapieansätze zum Tragen. Für den mittlerweile eher seltenen Fall, dass Temozolomid noch nicht in der Primärtherapie eingesetzt wurde, kann die Substanz als Standard der Rezidivtherapie angesehen werden. Zudem ist eine erneute Therapie mit Temozolomid in Erwägung zu ziehen, wenn es zu einem Tumorrezidiv nach einem längeren therapiefreien Intervall kommt. Die verfügbaren Daten legen nahe, dass die verschiedenen Dosisintensivierten Temozolomidschemata dem klassischen 5/28-Schema nicht überlegen sind. Von Temozolomid scheinen zudem hauptsächlich Patienten mit einem Rezidiv zu profitieren, deren Tumor einen methylierten MGMT-Promotor aufweist [11].

Alternativ zu Temozolomid kommen verschiedene Nitrosoharnstoffe zur Anwendung. In vielen Fällen wird einer Monotherapie mit Lomustin (CCNU) der Vorzug gegeben, meist in einer Dosis von 90-110 mg/m² alle 6-8 Wochen. Die in der Vergangenheit alternativ eingesetzten Nimustin (ACNU) und Carmustin (BCNU) kommen kaum noch zur Anwendung und stehen nur noch eingeschränkt zur Verfügung. BCNU kann in Form der „wafer“ auch lokal in der Resektionshöhle benutzt werden. Seit der Einführung von Temozolomid in der Primärtherapie ist der Einsatz von BCNU-Wafern jedoch kaum mehr üblich.

Im Gegensatz zu den USA und der Schweiz, wo eine Zulassung für Bevacizumab bei Patienten mit einem Glioblastom-Rezidiv besteht, liegt in Deutschland und den übrigen EUStaaten keine Zulassung vor. Während Bevacizumab, nicht zuletzt aufgrund seines ausgeprägten antiödematösen Effekts, teilweise zu einem deutlichen Rückgang klinischer Symptome führen kann, ist der Effekt des Präparats auf progressionsfreies Überleben und Gesamtüberleben in der Rezidivsituation nicht belegt. Daten aus einer randomisierten niederländischen Studie legen nahe, dass die Kombination von Bevacizumab mit dem 
Alkylans Lomustin einer alleinigen Bevacizumab- oder Lomustin-Behandlung überlegen ist [12]. Diese Frage wird im Rahmen der EORTC 26101-Studie definitiv beantwortet werden (NCT01290939).

\section{Ältere Glioblastom-Patienten}

Bei Patienten, die älter als 65-70 Jahre sind, ist der Stellenwert der kombinierten Radiochemotherapie in der Primärtherapie umstritten. Grundsätzlich profitieren ältere Patienten mit Glioblastomen weniger von der Behandlung als jüngere Patienten, die eine vergleichbare Therapie erhalten [13]. Die prognostisch günstigen Mutationen der IDH-Gene sind bei Gliomen älterer Patienten nicht vorhanden [14]. Das regelmässige Auftreten von Komorbiditäten und der bei älteren Patienten häufiger eingeschränkte Allgemeinzustand erfordern separate Behandlungsansätze für diese Patientenpopulation. Zwei randomisierte Phase III-Studien haben vor diesem Hintergrund die Wirksamkeit von Strahlentherapie alleine im Vergleich zur alleinigen Chemotherapie mit Temozolomid bei älteren Glioblastompatienten untersucht $[15,16]$. Die Ergebnisse beider Studien deuten darauf hin, dass bei Patienten, deren Tumor einen methylierten MGMT-Promoter aufweist, eine Chemotherapie mit Temozolomid einer Strahlentherapie überlegen ist. Umgekehrt profitieren Patienten mit unmethyliertem MGMT-Promoter mehr von einer Strahlentherapie. Ob die kombinierte Temozolomid-basierte Strahlentherapie gegenüber der alleinigen Bestrahlung bei älteren Patienten einen Vorteil bringt, wird in der zwischenzeitlich geschlossenen EORTC 26062-Studie untersucht. Ergebnisse werden im Laufe des Jahres 2015 erwartet.

Die Rolle von Bevacizumab bei älteren Patienten ist noch nicht abschliessend geklärt. Initiale Daten, wonach eine antiangiogene Therapie bei älteren Patienten möglicherweise besonders vielversprechend ist [17], konnten in den Subgruppenanalysen der RTOG 0825 und AVAGLIO-Studie zunächst nicht belegt werden [8, 9]. Die Kombination von Strahlentherapie und Bevacizumab wird in der Schweiz aktuell im Rahmen einer randomisierten Phase II- 
Studie bei Patienten über 65 Jahren untersucht (NCT01443676).

\section{Anaplastische Gliome (WHO-Grad III)}

Die Behandlung der anaplastischen Gliome war in den letzten Jahren einem Wandel unterworfen, der insbesondere durch ein besseres Verständnis des molekularen Profils dieser Tumoren zustande kam. Im Anschluss an die Operation war für lange Zeit die Strahlentherapie der Behandlungsstandard. Die Ergebnisse der NOA-04-Studie zeigten jedoch, dass eine Chemotherapie mit Temozolomid oder der Kombination von Procarbazin, CCNU und Vincristin (PCV) einer alleinigen Strahlentherapie gegenüber gleichwertig ist [18]. Die aktuell laufende CATNON-Studie (NCT00626990) untersucht die Frage, ob die konkomitante, adjuvante oder konkomitante und adjuvante Therapie mit Temozolomid zusätzlich zur Strahlentherapie einer alleinigen Bestrahlung bei anaplastischen Gliomen ohne $1 \mathrm{p} / 19 \mathrm{q}-$ Verlust überlegen ist.

Nachdem schon lange bekannt war, dass Tumoren mit oligodendroglialer Histologie besonders sensibel gegenüber einer Chemotherapie sind, wurde im Laufe der letzten Jahr klar, dass die „Chemosensitivität“ weniger durch den histologischen Subtyp als vielmehr durch das Vorhandensein einer 1p/19q-Kodeletion determiniert wird. Die Langzeitauswertung zweier grosser Studien zu Patienten mit anaplastischen oligodendroglialen Tumoren zeigen, dass die Kombination aus Strahlentherapie und Chemotherapie einer alleinigen Strahlentherapie bei Patienten mit 1p/19q-kodeletieren Tumoren in Bezug auf das Gesamtüberleben überlegen ist $[19,20]$. In beiden Studien, die in den 1990er Jahren durchgeführt wurden, erfolgte eine Kombination aus Bestrahlung und PCV-Chemotherapie. Ob gleichgute Ergebnisse mit dem in den letzten 10 Jahren wesentlich häufiger eingesetzten und besser verträglichen Temozolomid erreicht worden wären, ist nicht gesichert. Unklar bleibt zum jetzigen Zeitpunkt auch, ob ein primärer Verzicht auf die Strahlentherapie bei diesen Patienten ohne Verlust an Überlebenszeit möglich ist. 
Ein Therapiestandard für Patienten mit Rezidiv eines anaplastischen Glioms existiert nicht. Patienten, die in der Primärtherapie nicht bestrahlt wurden bzw. keine Chemotherapie erhalten haben, können die entsprechende Therapiemodalität im Rezidiv erhalten. Im Übrigen gelten ähnliche Überlegungen wie bei Patienten mit einem Glioblastomrezidiv.

\section{Niedriggradige Gliome}

Im Erwachsenenalter zählen astrozytäre, oligodendrogliale und gemischte oligoastrozytäre Tumoren zu den niedriggradigen Gliomen. Abgesehen von der Resektion ist der Nutzen anderer Therapieoptionen umstritten. Während für die Strahlentherapie eine Verlängerung des progressionsfreien Überlebens belegt ist, jedoch mutmasslich kein Effekt auf das Gesamtüberleben erwartet werden kann [21], ist die Rolle der Chemotherapie bisher weniger gut untersucht. Klassischerweise kommt die Chemotherapie im Rezidiv nach Ausschöpfung der lokalen Therapieoptionen zum Einsatz. Verwendet werden in erster Linie Temozolomid und zunehmend seltener Nitrosoharnstoffe oder das PCV-Schema. In der EORTC 2203326033-Studie, die die Strahlentherapie mit Temozolomidbehandlung verglich, zeigte sich ein Trend zur Überlegenheit der Strahlentherapie hinsichtlich des progressionsfreien Überlebens [22]. Aufgrund des begrenzten Nachbeobachtungszeitraums sind diese Daten jedoch nur als präliminär zu betrachten. Die RTOG 9802-Studie verglich die alleinige Strahlentherapie mit der Kombination aus Bestrahlung und PCV-Chemotherapie. Die bisher vorliegenden Auswertungen deuten darauf hin, dass die Hinzunahme der Chemotherapie zu einem verlängerten progressionsfreien Überleben und auch Gesamtüberleben führt [23]. Unklar bleibt die Bedeutung des molekularen Tumorprofils, insbesondere der Einfluss von IDHMutationsstatus, 1p/19q-Status und MGMT-Promotormethylierung auf den Effekt der zusätzlichen Chemotherapie. Diesbezüglich bleibt abzuwarten, ob weitere Analysen in der Zukunft eine Stratifizierung basierend auf einem oder mehreren molekularen Parametern möglich machen. 


\section{Gliomatosis cerebri}

Für die Diagnose einer Gliomatosis cerebri wird neben der histologische Diagnose eines glialen Tumors der bildgebende Nachweis einer Tumorausbreitung in mindestens 3 Hirnlappen gefordert. Aufgrund des ausgedehnten Tumorvolumens sind lokale Therapieformen nur eingeschränkt hilfreich bzw. im Falle der Strahlentherapie mit einem hohen Risiko für Neurotoxizität verbunden. Entsprechend wird bei vielen Patienten initial ein chemotherapeutischer Behandlungsansatz gewählt. Ergebnisse der nicht-randomisierten NOA-05-Studie ergaben ein medianes progressionsfreies Überleben von 14 Monaten mit der Kombination aus Procarbazin und CCNU [24]. Es wird angenommen, dass Temozolomid den Nitrosoharnstoff-haltigen Protokollen gleichwertig ist.

\section{Subependymale Riesenzellastrozytome (SEGA)}

SEGA sind seltene Tumoren, die insbesondere bei der tuberösen Sklerose auftreten. Nachdem lange Zeit ausschliesslich die chirugische Resektion etabliert war, besteht für Patienten mit nicht-resezierbaren SEGA mittlerweile eine zugelassene Therapie mit dem mTOR-Inhibitor Everolimus [25]. Rationale für diese Therapie ist die Aktivierung des mTOR-Pfadwegs, wenn der inhibitorische Einfluss der TSC1/2-Proteine entfällt.

\section{Ependymome}

Ependymome aller WHO-Grade werden nach Möglichkeit primär operiert. Anaplastische Ependymome (WHO-Grad III) werden in der Regel mit einer adjuvanten Strahlentherapie behandelt. Auch im Rezidivfall stellen die Resektion und ggf. die erneute Bestrahlung die ersten Therapieoptionen dar. Patienten mit einem Ependymom, bei dem die lokalen Therapieformen ausgeschöpft sind, stellen eine besondere therapeutische Herausforderung dar. Daten, die die Aktivität der Chemotherapie gegen Ependymome im Erwachsenenalter 
belegen, fehlen. Die Kombination von Carboplatin mit Bevacizumab wird derzeit in einer laufenden klinischen Studie getestet (NCT01295944). Der Tyrosinkinaseinhibitor Lapatinib wurde im Rahmen einer mittlerweile geschlossenen Phase II-Studie bei Patienten mit rezidiviertem Ependymom untersucht. Präleminäre Daten legen eine gewisse Wirksamkeit nahe, endgültige Ergebnisse liegen jedoch noch nicht vor [26].

\section{Embryonale Tumoren}

$\mathrm{Zu}$ den embryonalen Tumoren zählen Medulloblastome und supratentorielle primitive neuroektodermale Tumoren (PNET). Sie treten insbesondere bei Kindern, Jugendlichen und jungen Erwachsenen auf. Standardmässig erfolgt nach der Resektion eine Bestrahlung der Neuroachse. Obwohl die Bedeutung der Chemotherapie im Erwachsenenalter nicht durch grosse Studien gesichert ist, legen die verfügbaren Daten nahe, dass Platin-haltige Protokolle bei Patienten mit T3b-T4 und M1-4 Erkrankung oder postoperativem Resttumor einen Überlebensvorteil bringen [27]. Im Rahmen der NOA-07-Studie erfolgte eine adjuvante Chemotherapie in der Primärtherapie bei Medulloblastomen im Erwachsenenalter mit Cisplatin, CCNU und Vincristin. Ergebnisse liegen jedoch noch nicht vor.

Die Behandlung im Rezidiv ist nicht standardisiert. Es werden u.a. Therapieversuche mit Hochdosis-Chemotherapie und autologer Stammzelltransplantation unternommen. Bei Patienten mit einem Medulloblastom vom sonic hedgehog (SHH)-Subtyp kann in klinischen Studien bzw. im Rahmen eines individuellen Heilversuchs eine Behandlung mit einem smoothened receptor (SMO)-Inhibitor, z.B. Vismodegib, versucht werden.

\section{Keimzelltumoren}

Die Gruppe der Keimzelltumoren umfasst Germinome, Dottersacktumoren, embryonale Karzinome, Chorionkarzinome, Teratome und gemischte Keimzelltumoren. Bei reinen Germinomen können mit alleiniger Strahlentherapie oder Strahlentherapie in Kombination 
mit Chemotherapie hohe Heilungsraten erzielt werden [28]. Auch bei nicht-germinomatösen Keimzelltumoren ist die Strahlentherapie, ggf. ergänzt durch eine Chemotherapie, die Behandlung der Wahl, ggf. in Anlehnung an das SIOP-CNS-GCT-II-Protokoll.

\section{Pinealisparenchymtumoren}

Zu den Pinealisparenchymtumoren gehören Pineozytome (WHO-Grad II), Pinealisparenchymtumoren intermediärer Differenzierung (WHO-Grad II und III) sowie Pineoblastome (WHO-Grad IV). Therapiestandard sind die Resektion und Strahlentherapie. Kontrollierte Studien zur Chemotherapie liegen nur für Kinder und Jugendliche vor. Nach Ausschöpfung der lokalen Therapieoptionen kann bei Erwachsenen im Rezidiv ein Therapieversuch mit Cisplatin-haltigen Protokollen unternommen werden [29]. Insbesondere junge Erwachsene können in Anlehnung an die HIT-2000 bzw. HIT-REZ-05-Studie behandelt werden.

\section{Meningeome}

Die Resektion stellt für Meningeome die Therapie der Wahl dar. Bei atypischen WHO-Grad II-Meningeomen, die inkomplett reseziert sind, und bei anaplastischen Meningeomen (WHOGrad III) erfolgt im Anschluss eine Strahlentherapie. Die Chemotherapie hat keinen gesicherten Stellenwert in der Behandlung von Meningeom-Patienten und kommt nur dann zum Einsatz, wenn im Rezidivfall keine lokalen Therapieoptionen mehr bestehen. Therapieversuche können z.B. mit Hydroxyharnstoff oder Platin-haltigen Protokollen unternommen werden. Studien, die eine Wirksamkeit dieser Substanzen belegen, fehlen jedoch. Möglicherweise profitieren einzelne Patienten von einer Behandlung mit Bevacizumab oder Sunitinib [30, 31]. Therapieversuche mit dem Somatostatin-RezeptorAgonisten Octreotid waren weitgehend erfolglos [32]. 


\section{Tumoren von Hirnnerven und peripheren Nerven}

Bei den Hirnnerven treten Schwannome insbesondere im Bereich des N. vestibularis auf. Therapiestandard ist die Resektion, bei inoperablen Befunden oder Tumorprogredienz die Strahlentherapie. Medikamente mit gesicherter Wirksamkeit existieren nicht. Bei einzelnen Patienten kann ein Therapieversuch mit Bevacizumab oder Lapatinib in Erwägung gezogen werden [33, 34]. Bei malignen peripheren Nervenscheidentumoren (MPNST) kann bei Versagen der lokalen Therapieoptionen ein Behandlungsversuch mit Protokollen wie bei Weichteilsarkomen unternommen werden [35].

\section{Primäre ZNS-Lymphome (PCNSL)}

Bei keinem anderen Hirntumor ist die Wertigkeit der Chemotherapie so unzweifelhaft belegt wie bei den PCNSL. Unbestritten ist die Wirksamkeit von hochdosiertem Methotrexat (MTX), das in der Regel in einer Dosis von mindestens $3 \mathrm{~g} / \mathrm{m}^{2}$ eingesetzt wird. Unklar bleibt dagegen, welche Substanzen zusammen mit MTX gegeben werden sollen, um Ansprechrate und Überleben zu verbessern. Präparate, die hierfür grundsätzlich in Frage kommen, sind AraC und Ifosfamid [36]. Die Kombination von MTX-basierter Chemotherapie und konsolidierender Ganzhirnbestrahlung verlängert das progressionsfreie Überleben, hat jedoch keinen Einfluss auf das Gesamtüberleben bei Patienten, die unter Chemotherapie eine Komplettremission erreicht haben [37]. Bei jüngeren Patienten werden zunehmend Hochdosis-Protokolle mit anschliessender autologer Stammzelltransplantation benutzt. Im Rezidiv kommen grundsätzlich dieselben Therapieüberlegungen zum Tragen, ein Therapiestandard existiert jedoch nicht. Die Behandlung älterer PCNSL-Patienten erfordert spezifische Überlegungen, welche an anderer Stelle ausführlich dargestellt sind [38].

\section{Hirnmetastasen}

Die Behandlung von Hirnmetastasen ist in erster Linie eine Domäne der Neurochirurgie und 
Strahlentherapie. Bezüglich der Kontroverse hinsichtlich einer fokalen Bestrahlung im Vergleich zur Ganzhirnbestrahlung wird auf die weiterführende Literatur verwiesen [39]. Eine spezifische chemotherapeutische Behandlung von Hirnmetastasen existiert nicht. $\mathrm{Zu}$ berücksichtigen sind neben möglichen Vortherapien und damit einhergehenden Resistenzen insbesondere Alter und Karnofsky-Index, die für die Prognose eine entscheidende Rolle spielen [40]. Die Wahl der eingesetzten Substanz richtet sich in erster Linie nach der histologischen Diagnose und weicht somit nicht von der Therapie von Metastasen ausserhalb des ZNS ab. Sofern bei eingeschränkter Datenlage beurteilbar, ist das Ansprechen von Hirnmetastasen auf eine chemotherapeutische Behandlung wahrscheinlich nicht wesentlich anders als das von Metastasen ausserhalb des ZNS [41]. Ein ZNS-spezifisches Problem stellt u.U. die Blut-Hirn-Schranke dar, welche das Erreichen suffizienter Konzentrationen von Chemotherapeutika im Parenchym verhindern kann. Da Metastasen im ZNS in den meisten Fällen jedoch Kontrastmittel-aufnehmend sind und somit keine intakte Blut-Hirn-Schranke besitzen, scheint dieser Resistenzmechanismus in der Regel nicht von entscheidender Bedeutung zu sein. Am meisten scheinen Patienten mit einem kleinzelligen Bronchialkarzinom [42] bzw. mit Keimzelltumoren von einer Chemotherapie zu profitieren. Für die am häufigsten in das ZNS metastasierenden Tumoren, u.a. nicht-kleinzellige Bronchialkarzinome, Mamma-Karzinome und Melanome, sind die Ansprechraten dagegen, ähnlich wie bei anderen systemischen Tumormanifestationen, häufig unbefriedigend. Typischerweise sind die Ansprechraten von ZNS-Metastasen auf eine Chemotherapie am höchsten, wenn zuvor keine Systemtherapie erfolgt war. Ob die Kombination von Strahlenund Chemotherapie einen Vorteil zur alleinigen Bestrahlung hat, ist nicht belegt [43]. Neben den klassischen Chemotherapeutika stehen für einige Tumorentitäten neue Therapieansätze mit „,small molecules“ bzw. Immuntherapeutika zur Verfügung. Bei Melanom-Patienten scheint der CTLA-4-Antikörper Ipilimumab auch gegen Hirnmetastasen zu wirken [44]. Inwiefern dies auch für die aktuell umfassend getesteten PD-1-Inhibitoren 
gilt, bleibt abzuwarten. Bei Melanom-Patienten, deren Tumor eine BRAF V600E-Mutation aufweist, können die entsprechenden BRAF-Inhibitoren Vemurafenib bzw. Dabrafenib mit gewissen Erfolgsaussichten eingesetzt werden [45]. Einige Patientinnen mit einem HER2positiven Mamma-Karzinom scheinen von einer Therapie mit Lapatinib zu profitieren [46].

\section{Meningeosis neoplastica}

Der Einsatz von Chemotherapeutika im Falle einer Tumordissemination in den Liquorraum muss im Kontext der zerebralen und systemischen Tumorlast betrachtet werden. Zudem entscheidet die Art der Tumormanifestation im Subarachnoidalraum über eine mögliche intrathekale Behandlung. Patienten mit soliden Tumorabsiedlungen werden in der Regel bestrahlt oder erhalten eine systemische Therapie, analog der von Metastasen im Hirnparenchym. Die intrathekale Therapie kommt dann zum Einsatz, wenn nicht-adhärente Tumorzellen im Liquor nachgewiesen werden. Zur Verfügung stehen dann insbesondere Methotrexat und Cytarabin, in Deutschland ist auch Thiotepa zugelassen. Cytarabin steht insbesondere zur Therapie hämatologischer Neoplasien auch in liposomaler Form (Depocyte ${ }^{\circledR}$ ) zur Verfügung und muss dann nur alle 14 Tage appliziert werden. Dem stehen vermehrte Nebenwirkungen, insbesondere ein gehäuftes Auftreten von Arachnoitiden, gegenüber. In Bezug auf das Überleben konnte für liposomales Cytarabin bisher kein eindeutiger Vorteil gegenüber anderen Substanzen belegt werden.

\section{Aktuelle Entwicklungen und experimentelle Ansätze in der medikamentösen}

\section{Tumortherapie in der Neuroonkologie}

Im Fokus der klinischen Entwicklung in der Neuroonkologie standen in den letzten Jahren insbesondere Präprate, die die Angiogenese hemmen sollen. Zumindest bei

Glioblastompatienten wurden die hohen Erwartungen in der Primärtherapie mit dem VEGFAntikörper Bevacizumab nicht erfüllt. Der VEGF-Rezeptorinhibitor Cediranib wurde ebenso 
wie der Integrin-Antagonist Cilengitide aufgrund negativer Studiendaten bei Patienten mit einem Glioblastomrezidiv nicht weiterentwickelt. Inhibitoren der Signaltransduktion über andere Rezeptortyrosinkinasen wie EGFR und PDGFR, die eine vermeintlich gezieltere Hemmung des Tumorwachstums erreichen sollen, sind bei hirneigenen Tumoren bis jetzt weitgehend wirkungslos geblieben und kommen in erster Linie bei Patienten mit Hirnmetastasen in Frage, bei welchen z.B. eine aktivierende EGFR-Mutation im Tumor nachgewiesen wird. Neuere Substanzen wie Sorafenib, Sunitinib oder weiterentwickelte EGFR-Inhibitoren wurden im Bereich der Neuroonkologie noch nicht umfangreich getestet. Aktuelle Entwicklungen der medikamentösen Behandlung von Hirntumoren umfassen insbesondere neue immunotherapeutische Strategien. Hierzu zählen bei den Glioblastomen verschiedene Vakzinierungsansätze, wobei der Peptid-Impfstoff Rindopepimut, der eine mutierte Variante des EGF-Rezeptors (EGFRvIII) nachahmt, aktuell in einer Phase III-Studie bei Glioblastom-Patienten untersucht wird (NCT01480479). Zudem wird eine Gruppe neuer Substanzen bei Patienten mit hirneigenen Tumoren und Metastasen getestet, die als „immune checkpoint"-Inhibitoren bezeichnet werden. Der CTLA-4-Antikörper Ipilimumab, der bereits für die Behandlung von Patienten mit metastasiertem malignem Melanom zugelassen ist, wird genauso wie Antikörper, die den PD-1-Signalweg hemmen, derzeit bei verschiedenen neuroonkologischen Tumorentitäten im Rahmen klinischer Studien getestet. Möglicherweise stellen diese Präparate in der Zukunft eine neue medikamentöse Behandlungsoption in der Neuroonkologie dar.

\section{Fazit für die Praxis}

Die Chemotherapie hat insbesondere in der Behandlung von Glioblastomen, anaplastischen Gliomen und ZNS-Lymphomen einen festen Stellenwert in der klinischen Neuroonkologie. Dies betrifft sowohl die Primär- als auch Rezidivtherapie. Bei anderen Tumorentitäten, insbesondere niedriggradigen Gliomen, Ependymomen und Hirnmetastasen muss der Einsatz 
der Chemotherapie im Kontext der Gesamtsituation und somit letztlich auf individueller Basis entschieden werden, da Daten aus grossen Studien fehlen. Für Meningeome und Schwannome stehen weiterhin keine überzeugenden medikamentösen Therapieoptionen zur Verfügung. Es ist davon auszugehen, dass neben den wichtigsten Therapie-unabhängigen prognostischen Faktoren sowie der Histologie zukünftig molekulare Marker eine noch grössere Bedeutung bei der Therapieentscheidung spielen werden. Zudem besteht insbesondere im Bereich der Glioblastome die Hoffnung, dass immuntherapeutische Behandlungsansätze das Stadium klinischer Studien verlassen werden und Einzug in die Standardtherapie finden werden. 


\section{Literatur}

1. Roth P, Wick W, Weller M (2010) Steroids in neurooncology: actions, indications, side-effects. Curr Opin Neurol 23:597-602.

2. Weller M, Stupp R, Wick W (2012) Epilepsy meets cancer: when, why, and what to do about it? Lancet Oncol 13:e375-382.

3. Weller M, Pfister SM, Wick W, Hegi ME, Reifenberger G, Stupp R (2013) Molecular neuro-oncology in clinical practice: a new horizon. Lancet Oncol 14:e370-379.

4. Wick W, Meisner C, Hentschel B, Platten M, Schilling A, Wiestler B, et al. (2013) Prognostic or predictive value of MGMT promoter methylation in gliomas depends on IDH1 mutation. Neurology 81:1515-1522.

5. Stupp R, Mason WP, van den Bent MJ, Weller M, Fisher B, Taphoorn MJ, et al. (2005) Radiotherapy plus concomitant and adjuvant temozolomide for glioblastoma. N Engl J Med 352:987-996.

6. Gilbert MR, Wang M, Aldape KD, Stupp R, Hegi ME, Jaeckle KA, et al. (2013) Dosedense temozolomide for newly diagnosed glioblastoma: a randomized phase III clinical trial. J Clin Oncol 31:4085-4091.

7. Hegi ME, Liu L, Herman JG, Stupp R, Wick W, Weller M, et al. (2008) Correlation of O6-methylguanine methyltransferase (MGMT) promoter methylation with clinical outcomes in glioblastoma and clinical strategies to modulate MGMT activity. J Clin Oncol 26:41894199.

8. Gilbert MR, Dignam JJ, Armstrong TS, Wefel JS, Blumenthal DT, Vogelbaum MA, et al. (2014) A randomized trial of bevacizumab for newly diagnosed glioblastoma. N Engl J Med 370:699-708.

9. Chinot OL, Wick W, Mason W, Henriksson R, Saran F, Nishikawa R, et al. (2014) Bevacizumab plus radiotherapy-temozolomide for newly diagnosed glioblastoma. N Engl J Med 370:709-722.

10. Stupp R, Hegi ME, Gorlia T, Erridge SC, Perry J, Hong YK, et al. (2014) Cilengitide combined with standard treatment for patients with newly diagnosed glioblastoma with methylated MGMT promoter (CENTRIC EORTC 26071-22072 study): a multicentre, randomised, open-label, phase 3 trial. Lancet Oncol 15:1100-1108.

11. Tabatabai G, Wick W, Steinbach JP, Wick A, Schnell O, Hau P, et al. (2014) MGMT promoter methylation as a prognostic biomarker for benefit from dose-intensified temozolomide rechallenge in progressive glioblastoma: First results from the randomized phase II DIRECTOR trial. J Clin Oncol 32:5s, (suppl; abstr 2015).

12. Taal W, Oosterkamp HM, Walenkamp AM, Dubbink HJ, Beerepoot LV, Hanse MC, et al. (2014) Single-agent bevacizumab or lomustine versus a combination of bevacizumab plus lomustine in patients with recurrent glioblastoma (BELOB trial): a randomised controlled phase 2 trial. Lancet Oncol 15:943-953.

13. Stupp R, Hegi ME, Mason WP, van den Bent MJ, Taphoorn MJ, Janzer RC, et al. (2009) Effects of radiotherapy with concomitant and adjuvant temozolomide versus radiotherapy alone on survival in glioblastoma in a randomised phase III study: 5-year analysis of the EORTC-NCIC trial. Lancet Oncol 10:459-466.

14. Hartmann C, Hentschel B, Wick W, Capper D, Felsberg J, Simon M, et al. (2010) 
Patients with IDH1 wild type anaplastic astrocytomas exhibit worse prognosis than IDH1mutated glioblastomas, and IDH1 mutation status accounts for the unfavorable prognostic effect of higher age: implications for classification of gliomas. Acta Neuropathol 120:707718 .

15. Wick W, Platten M, Meisner C, Felsberg J, Tabatabai G, Simon M, et al. (2012) Temozolomide chemotherapy alone versus radiotherapy alone for malignant astrocytoma in the elderly: the NOA-08 randomised, phase 3 trial. Lancet Oncol 13:707-715.

16. Malmstrom A, Gronberg BH, Marosi C, Stupp R, Frappaz D, Schultz H, et al. (2012) Temozolomide versus standard 6-week radiotherapy versus hypofractionated radiotherapy in patients older than 60 years with glioblastoma: the Nordic randomised, phase 3 trial. Lancet Oncol 13:916-926.

17. Kreisl TN, Zhang W, Odia Y, Shih JH, Butman JA, Hammoud D, et al. (2011) A phase II trial of single-agent bevacizumab in patients with recurrent anaplastic glioma. Neuro Oncol 13:1143-1150.

18. Wick W, Hartmann C, Engel C, Stoffels M, Felsberg J, Stockhammer F, et al. (2009) NOA-04 randomized phase III trial of sequential radiochemotherapy of anaplastic glioma with procarbazine, lomustine, and vincristine or temozolomide. J Clin Oncol 27:5874-5880.

19. Cairncross G, Wang M, Shaw E, Jenkins R, Brachman D, Buckner J, et al. (2013) Phase III trial of chemoradiotherapy for anaplastic oligodendroglioma: long-term results of RTOG 9402. J Clin Oncol 31:337-343.

20. van den Bent MJ, Brandes AA, Taphoorn MJ, Kros JM, Kouwenhoven MC, Delattre JY, et al. (2013) Adjuvant procarbazine, lomustine, and vincristine chemotherapy in newly diagnosed anaplastic oligodendroglioma: long-term follow-up of EORTC brain tumor group study 26951. J Clin Oncol 31:344-350.

21. van den Bent MJ, Afra D, de Witte O, Ben Hassel M, Schraub S, Hoang-Xuan K, et al. (2005) Long-term efficacy of early versus delayed radiotherapy for low-grade astrocytoma and oligodendroglioma in adults: the EORTC 22845 randomised trial. Lancet 366:985-990.

22. Baumert BG, Mason WP, Ryan G, Bromberg JE, Van Den Bent MJ, Hoang-Xuan K, et al. (2013) Temozolomide chemotherapy versus radiotherapy in molecularly characterized (1p loss) low-grade glioma: A randomized phase III intergroup study by the EORTC/NCICCTG/TROG/MRC-CTU (EORTC 22033-26033). J Clin Oncol 31, (suppl; abstr 2007).

23. Buckner JC, Pugh SL, Shaw EG, Gilbert MR, Barger G, Coons S, et al. (2014) Phase III study of radiation therapy (RT) with or without procarbazine, CCNU, and vincristine (PCV) in low-grade glioma: RTOG 9802 with Alliance, ECOG, and SWOG. J Clin Oncol 32:5s, (suppl; abstr 2000).

24. Glas M, Bahr O, Felsberg J, Rasch K, Wiewrodt D, Schabet M, et al. (2011) NOA-05 phase 2 trial of procarbazine and lomustine therapy in gliomatosis cerebri. Ann Neurol 70:445-453.

25. Franz DN, Belousova E, Sparagana S, Bebin EM, Frost M, Kuperman R, et al. (2014) Everolimus for subependymal giant cell astrocytoma in patients with tuberous sclerosis complex: 2-year open-label extension of the randomised EXIST-1 study. Lancet Oncol 15:1513-1520.

26. Gilbert M, Yuan Y, Wani K, Wu J, Omuro A, Lieberman F, et al. (2014) A phase II study of lapatinib and dose-dense temozolomide (TMZ) for adults with recurrent ependymoma: a CERN clinical trial. Neuro Oncol 16 (suppl 5): v13. 
27. Brandes AA, Franceschi E, Tosoni A, Blatt V, Ermani M (2007) Long-term results of a prospective study on the treatment of medulloblastoma in adults. Cancer 110:2035-2041.

28. Jensen AW, Laack NN, Buckner JC, Schomberg PJ, Wetmore CJ, Brown PD (2010) Long-term follow-up of dose-adapted and reduced-field radiotherapy with or without chemotherapy for central nervous system germinoma. Int J Radiat Oncol Biol Phys 77:14491456.

29. Galanis E, Buckner JC, Schomberg PJ, Hammack JE, Raffel C, Scheithauer BW (1997) Effective chemotherapy for advanced CNS embryonal tumors in adults. J Clin Oncol 15:2939-2944.

30. Nayak L, Iwamoto FM, Rudnick JD, Norden AD, Lee EQ, Drappatz J, et al. (2012) Atypical and anaplastic meningiomas treated with bevacizumab. J Neurooncol 109:187-193.

31. Kaley TJ, Wen P, Schiff D, Ligon K, Haidar S, Karimi S, et al. (2014) Phase II trial of sunitinib for recurrent and progressive atypical and anaplastic meningioma. Neuro Oncol.

32. Johnson DR, Kimmel DW, Burch PA, Cascino TL, Giannini C, Wu W, et al. (2011) Phase II study of subcutaneous octreotide in adults with recurrent or progressive meningioma and meningeal hemangiopericytoma. Neuro Oncol 13:530-535.

33. Plotkin SR, Stemmer-Rachamimov AO, Barker FG, 2nd, Halpin C, Padera TP, Tyrrell A, et al. (2009) Hearing improvement after bevacizumab in patients with neurofibromatosis type 2. N Engl J Med 361:358-367.

34. Karajannis MA, Legault G, Hagiwara M, Ballas MS, Brown K, Nusbaum AO, et al. (2012) Phase II trial of lapatinib in adult and pediatric patients with neurofibromatosis type 2 and progressive vestibular schwannomas. Neuro Oncol 14:1163-1170.

35. Grobmyer SR, Reith JD, Shahlaee A, Bush CH, Hochwald SN (2008) Malignant Peripheral Nerve Sheath Tumor: molecular pathogenesis and current management considerations. Journal of surgical oncology 97:340-349.

36. Korfel A, Schlegel U (2013) Diagnosis and treatment of primary CNS lymphoma. Nat Rev Neurol 9:317-327.

37. Thiel E, Korfel A, Martus P, Kanz L, Griesinger F, Rauch M, et al. (2010) High-dose methotrexate with or without whole brain radiotherapy for primary CNS lymphoma (GPCNSL-SG-1): a phase 3, randomised, non-inferiority trial. Lancet Oncol 11:1036-1047.

38. Roth P, Hoang-Xuan K (2014) Challenges in the treatment of elderly patients with primary central nervous system lymphoma. Curr Opin Neurol 27:697-701.

39. Kocher M, Soffietti R, Abacioglu U, Villa S, Fauchon F, Baumert BG, et al. (2011) Adjuvant whole-brain radiotherapy versus observation after radiosurgery or surgical resection of one to three cerebral metastases: results of the EORTC 22952-26001 study. J Clin Oncol 29:134-141.

40. Sperduto PW, Kased N, Roberge D, Xu Z, Shanley R, Luo X, et al. (2012) Effect of tumor subtype on survival and the graded prognostic assessment for patients with breast cancer and brain metastases. Int J Radiat Oncol Biol Phys 82:2111-2117.

41. Owonikoko TK, Arbiser J, Zelnak A, Shu HK, Shim H, Robin AM, et al. (2014) Current approaches to the treatment of metastatic brain tumours. Nat Rev Clin Oncol 11:203222.

42. Chen G, Huynh M, Fehrenbacher L, West H, Lara PN, Jr., Yavorkovsky LL, et al. (2009) Phase II trial of irinotecan and carboplatin for extensive or relapsed small-cell lung 
cancer. J Clin Oncol 27:1401-1404.

43. Sperduto PW, Wang M, Robins HI, Schell MC, Werner-Wasik M, Komaki R, et al. (2013) A phase 3 trial of whole brain radiation therapy and stereotactic radiosurgery alone versus WBRT and SRS with temozolomide or erlotinib for non-small cell lung cancer and 1 to 3 brain metastases: Radiation Therapy Oncology Group 0320. Int J Radiat Oncol Biol Phys $85: 1312-1318$.

44. Margolin K, Ernstoff MS, Hamid O, Lawrence D, McDermott D, Puzanov I, et al. (2012) Ipilimumab in patients with melanoma and brain metastases: an open-label, phase 2 trial. Lancet Oncol 13:459-465.

45. Falchook GS, Long GV, Kurzrock R, Kim KB, Arkenau TH, Brown MP, et al. (2012) Dabrafenib in patients with melanoma, untreated brain metastases, and other solid tumours: a phase 1 dose-escalation trial. Lancet 379:1893-1901.

46. Lin NU, Carey LA, Liu MC, Younger J, Come SE, Ewend M, et al. (2008) Phase II trial of lapatinib for brain metastases in patients with human epidermal growth factor receptor 2-positive breast cancer. J Clin Oncol 26:1993-1999. 
Tabelle 1: Medikamentöse Therapie in der Neuroonkologie

\begin{tabular}{|c|c|c|c|}
\hline Substanz & $\begin{array}{l}\text { Applikations- } \\
\text { modus }\end{array}$ & Wirkmechanismus & Indikation \\
\hline \multicolumn{4}{|l|}{$\begin{array}{l}\text { Klassische } \\
\text { Chemotherapeutika }\end{array}$} \\
\hline Nitrosoharnstoffe & & & Gliome \\
\hline ACNU (Nimustin) & i.v. & DNA-Alkylierung & \\
\hline BCNU (Carmustin) & i.v. & & \\
\hline $\mathrm{BCNU}$ & Interstitiell & & \\
\hline CCNU (Lomustin) & p.o. & & \\
\hline Temozolomid & p.o. & DNA-Methylierung & Gliome \\
\hline Procarbazin & p.o. & DNA-Methylierung & Gliome \\
\hline Hydroxyharnstoff & p.o. & $\begin{array}{l}\text { antiproliferativ, Hemmung der } \\
\text { Ribonukleosid- } \\
\text { bisphosphatreduktase }\end{array}$ & Meningeome \\
\hline Topotecan, Irinotecan & i.v. & Topoisomerase I-Hemmung & \begin{tabular}{|l|} 
Gliome \\
Metastasen
\end{tabular} \\
\hline Etoposid & p.o., i.v. & Topoisomerase II-Hemmung & $\begin{array}{l}\text { Gliome } \\
\text { Diverse }\end{array}$ \\
\hline (Teniposid) & i.v. & & Gliome \\
\hline Vincristin & i.v. & Mitosehemmung & $\begin{array}{l}\text { Gliome } \\
\text { Medulloblastome } \\
\text { PCNSL } \\
\end{array}$ \\
\hline Carboplatin & i.v. & DNA-Schädigung & Gliome \\
\hline $\begin{array}{l}\text { Signaltransduktions- } \\
\text { hemmung }\end{array}$ & & & \\
\hline $\begin{array}{l}\text { Imatinib, Gefitinib, } \\
\text { Erlotinib; Lapatinib } \\
\text { u.a. }\end{array}$ & p.o. & $\begin{array}{l}\text { Hemmung der } \\
\text { Signaltransduktion über } \\
\text { Tyrosinkinaserezeptoren } \\
\end{array}$ & $\begin{array}{l}\text { Gliome } \\
\text { Hirnmetastasen }\end{array}$ \\
\hline $\begin{array}{l}\text { Rapamycin (oral)/ } \\
\text { Temsirolimus (i.v.) } \\
\text { Everolimus }\end{array}$ & $\begin{array}{l}\text { p.o./ i.v. } \\
\text { p.o. }\end{array}$ & mTOR-Hemmung & $\begin{array}{l}\text { Gliome } \\
\text { Subependymale } \\
\text { Riesenzellastrozy } \\
\text { tome }\end{array}$ \\
\hline $\begin{array}{l}\text { Vemurafenib, } \\
\text { Dabrafenib }\end{array}$ & p.o. & $\begin{array}{l}\text { BRAF-Inhibition (bei Vorliegen } \\
\text { einer V600E-Mutation) }\end{array}$ & $\begin{array}{l}\text { Hirnmetastasen, } \\
\text { Gliome } \\
\text { (experimentell) }\end{array}$ \\
\hline
\end{tabular}




\begin{tabular}{|l|l|l|l|}
\hline & p.o. & SMO-Hemmung & $\begin{array}{l}\text { Medulloblastome } \\
\text { vom SHH-Typ }\end{array}$ \\
\hline $\begin{array}{l}\text { Angiogenese- } \\
\text { hemmung }\end{array}$ & & & $\begin{array}{l}\text { Hirnmetastasen/ } \\
\text { experimentell }\end{array}$ \\
\hline $\begin{array}{l}\text { Sorafenib, Sunitinib } \\
\text { u.a. }\end{array}$ & p.o. & $\begin{array}{l}\text { Hemmung der } \\
\text { Signaltransduktion } \\
\text { (verschiedene Rezeptor- } \\
\text { Tyrosinkinasen) }\end{array}$ & $\begin{array}{l}\text { Vliome (keine } \\
\text { Zulassung in } \\
\text { Deutschland) }\end{array}$ \\
\hline Bevacizumab & i.v. & VEGF-Antikörper & $\begin{array}{l}\text { Glioblastome } \\
\text { (experimentell) }\end{array}$ \\
\hline Immuntherapeutika & intradermal & EGFRvIII-Vakzine & $\begin{array}{l}\text { Hirnmetastasen, } \\
\text { Gliome } \\
\text { (experimentell) }\end{array}$ \\
\hline Rindopepimut & i.v. & CTLA-4-Inhibition & $\begin{array}{l}\text { Hirnmetastasen, } \\
\text { (experimentell) }\end{array}$ \\
\hline Ipilimumab & i.v. & PD-1-Inhibition & \\
\hline $\begin{array}{l}\text { Nivolumab, } \\
\text { Pembrolizumab }\end{array}$ & & & \\
\hline
\end{tabular}




\section{Multiple choice-Fragen}

\begin{tabular}{|c|c|}
\hline 1. & Welche Aussage zur Chemotherapie von Glioblastomen ist richtig? \\
\hline A & Bei jüngeren Patienten besteht die Standardtherapie aus einer alleinigen Bestrahlung. \\
\hline B & $\begin{array}{l}\text { Bei älteren Patienten kann die Bestimmung des MGMT-Status zur } \\
\text { Therapiestratifizierung herangezogen werden. }\end{array}$ \\
\hline $\mathrm{C}$ & Das Resektionsausmass bestimmt die Art der adjuvanten Therapie. \\
\hline $\mathrm{D}$ & Der 1p/19q-Status bestimmt die Wahl des Chemotherapeutikums. \\
\hline E & Nitrosoharnstoffe kommen nur lokal in der Resektionshöhle zum Einsatz. \\
\hline 2. & Welche Aussage zur Behandlung von anaplastischen Gliomen ist richtig? \\
\hline$\vec{A}$ & $\begin{array}{l}\text { Nach einer makroskopischen Komplettresektion ist keine adjuvante Therapie } \\
\text { notwendig. }\end{array}$ \\
\hline B & Die Therapie sollte immer analog der von Glioblastomen erfolgen. \\
\hline $\mathrm{C}$ & Die Standardchemotherapie besteht aus Platin-haltigen Protokollen. \\
\hline $\mathrm{D}$ & $\begin{array}{l}\text { Die Bestimmung des 1p/19q-Status kann bei der Therapieentscheidung } \\
\text { richtungsweisend sein. }\end{array}$ \\
\hline E & Das PCV-Regime ist in der Regel nebenwirkungsfrei. \\
\hline 3. & $\begin{array}{l}\text { Welche Empfehlung sprechen Sie bei der Behandlung von Patienten mit } \\
\text { niedriggradigen Gliomen aus? }\end{array}$ \\
\hline A & Alle Patienten sollten mit einer Chemotherapie behandelt werden. \\
\hline B & Alle Patienten sollten im Anschluss an die Resektion bestrahlt werden. \\
\hline $\mathrm{C}$ & $\begin{array}{l}\text { Die Bestimmung molekularer Marker ist noch nicht Teil der klinischen Routine, kann } \\
\text { aber für die Therapieentscheidung hilfreich sein. }\end{array}$ \\
\hline $\mathrm{D}$ & Im Rezidiv sollte grundsätzlich keine erneute Resektion versucht werden. \\
\hline $\mathrm{E}$ & Im Fall einer Chemotherapie sollte in erster Linie Etoposid verwendet werden. \\
\hline 4. & $\begin{array}{l}\text { Welche Aussage zum Einsatz von Bevacizumab bei Patienten mit einem } \\
\text { Glioblastom ist richtig? }\end{array}$ \\
\hline A & Bevacizumab wirkt als Antikörper gegen EGFR. \\
\hline B & Bevacizumab ist weltweit zur Glioblastom-Behandlung zugelassen. \\
\hline $\mathrm{C}$ & $\begin{array}{l}\text { Die Hinzunahme von Bevacizumab zur Temozolomid-basierten Radiochemotherapie } \\
\text { verlängert das progressionsfreie Überleben. }\end{array}$ \\
\hline $\mathrm{D}$ & $\begin{array}{l}\text { Die Hinzunahme von Bevacizumab zur Temozolomid-basierten Radiochemotherapie } \\
\text { verlängert das Gesamtüberleben. }\end{array}$ \\
\hline E & $\begin{array}{l}\text { Typische Nebenwirkungen von Bevacizumab sind Übelkeit, Erbrechen und } \\
\text { Myelosuppression. }\end{array}$ \\
\hline 5. & Welche Aussage zu subependymalen Riesenzellastrozytomen ist richtig? \\
\hline $\mathrm{A}$ & Es handelt sich um die häufigsten glialen Tumoren. \\
\hline B & Die Strahlentherapie ist die Behandlung der Wahl. \\
\hline $\mathrm{C}$ & Riesenzellastrozytome kommen v.a. bei der Neurofibromatose vor. \\
\hline $\mathrm{D}$ & Maligne Entartungen sind die Regel. \\
\hline E & $\begin{array}{l}\text { Mit dem mTOR-Inhibitor Everolimus besteht seit wenigen Jahren eine zugelassene } \\
\text { medikamentöse Behandlungsoption. }\end{array}$ \\
\hline 6. & Welche Aussage zu Medulloblastomen im Erwachsenenalter ist richtig? \\
\hline A & Medulloblastome sollten grundsätzlich nicht reseziert sondern nur biopsiert werden. \\
\hline
\end{tabular}




\begin{tabular}{|l|l|}
\hline B & Der Nutzen der Strahlentherapie ist noch nicht definitiv belegt. \\
\hline C & $\begin{array}{l}\text { Die medikamentöse Behandlung sollte immer aus einer Hochdosis-Chemotherapie und } \\
\text { autologer Stammzelltransplantation bestehen. }\end{array}$ \\
\hline D & $\begin{array}{l}\text { Patienten mit einem Medulloblastom vom SHH-Subtyp können experimentell mit } \\
\text { einem SMO-Inhibitor behandelt werden. }\end{array}$ \\
\hline E & Medulloblastome kommen vorwiegend bei älteren Patienten vor. \\
\hline & \\
\hline 7. & Was empfehlen Sie bei der Behandlung von primären ZNS-Lymphomen? \\
\hline A & Bei allen Patienten sollte eine Komplettresektion des Tumors angestrebt werden. \\
\hline B & Eine Strahlentherapie ist bei allen Patienten indiziert. \\
\hline C & $\begin{array}{l}\text { Bei Fehlen von Kontraindikationen ist die MTX-basierte Chemotherapie die Therapie } \\
\text { der Wahl. }\end{array}$ \\
\hline D & Eine intrathekale Chemotherapie sollte immer durchgeführt werden. \\
\hline E & Ältere Patienten sollten möglichst nicht mit MTX behandelt werden. \\
\hline & \\
\hline 8. & Bei welcher Tumorentität ist die Chemotherapie die wichtigste Therapieform? \\
\hline A & Ependymome \\
\hline B & Schwannome \\
\hline C & Meningeome \\
\hline D & Primäre ZNS-Lymphome \\
\hline E & Anaplastische Gliome ohne 1p/19q-Kodeletion \\
\hline & \\
\hline 9. & Welche Aussage zur intrathekalen Chemotherapie trifft zu? \\
\hline A & Bei primären ZNS-Lymphomen ist die intrathekale Therapie grundsätzlich indiziert. \\
\hline B & Die lumbale Applikation ist der Verabreichung über eine Reservoir gleichwertig. \\
\hline C & $\begin{array}{l}\text { Patienten mit soliden Hirnmetastasen und einer Meningeosis neoplastica sollten immer } \\
\text { auch intrathekal behandelt werden. }\end{array}$ \\
\hline D & Die intrathekale Chemotherapie kann nie zu einer Myelosuppression führen. \\
\hline E & $\begin{array}{l}\text { MTX stellt bei der Meningeosis der meisten soliden Tumoren den Therapiestandard } \\
\text { dar. }\end{array}$ \\
\hline & \\
\hline $\mathbf{1 0 .}$ & Welches Substanz ist für eine intrathekale Therapie zugelassen? \\
\hline A & Temozolomid \\
\hline B & Carboplatin \\
\hline C & Cytarabin \\
\hline D & Vincristin \\
\hline E & Etoposid \\
\hline & \\
\hline
\end{tabular}

Lösungen: $1 B-2 D-3 C-4 C-5 E-6 D-7 C-8 D-9 E-10 C$ 